\title{
Back to Basics
}

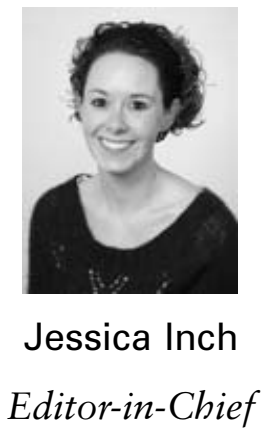

$\mathrm{W}$ elcome to the new look for 2008. Things are set to develop within the Journal this year and we hope you will join in. We want this to be a year of education, bringing the emphasis back to evidence based learning. To enable this, we have added a few new features, one of which is a 'Drug Profile', which is aimed at refreshing our memories with the basic characteristics of the drugs we use the most in our speciality. The Journal will also now include 'unit profiles', which allow you to tell us about your anaesthetic/recovery units, how they are run, the unique challenges you face and what you like most about your units. This will allow us to share management ideas and strategies. To tell us about your unit, go to www.barna.co.uk and download the questionnaire and return them to the Editorial team, along with any photos you wish to send.

So, this is a call to all our members to put pen to paper, crank up the PC and get writing! Just to encourage you, we have drawn up a list of topics which you may have an interest in, or have already written about. Either way, please contact the Editorial team. They will help you prepare your submission for publication.

- Patient case studies: These can be from any surgical speciality, i.e. special needs to anaesthesia for the cardiac patient.

- Managing a specific clinical complication in anaesthetics/recovery: For example, residual paralysis, aspiration, pulmonary oedema, arrhythmia, hypovolamia, hypothermia, neurological deficit, TURP syndrome, intractable PONV, pain, perioperative ischaemia, etc.

- Management issues: We want to know about the problems you experience in your units/theatres and how you overcome them. These can be staffing issues, skill mix, educational provisions in your unit, handling throughput overload, infection control, parents in recovery and conflict issues in the multidisciplinary team.

- Quality and audit: Reports on any audit you have been involved in and why was it necessary. What was audit tool? What did the results show? What action was taken?

- Ethical dilemmas: Write about any professional/ ethical/legal situation, issue or dilemma you have experienced in your anaesthetic or recovery unit. This could be presented as a reflection using a recognised model. We want to hear about issues surrounding advocacy, accountability, delegation, consent, dignity, confidentiality and negligence. These are all of great interest to your fellow members.

- Reflection: What incident has caused you to stop and think 'What happened there?' Use any reflective model, i.e. Gibbs or Johns.

- Information about your unit: We would like to hear about your unit. What is your speciality and staffing requirements? What type of training opportunities do you offer and what makes you enjoy working there? Download the questionnaire at www.barna. co.uk. 
- Conference and study day reports: Have you attended a conference or a study day? What did you think? Would you recommend it?

- Mentoring and assessing: How do you organise students and new staff in your anaesthetic/recovery? Do you have any examples of good mentorship, assessment or technique? What orientation or teaching packages do you have in place?

- Send examples of discharge criteria, protocols, guidelines or standards: Include rationale about why they are effective and how other units could learn from them.
The above are just a few examples of topics that would be of great interest to the Association. If you do not feel confident to present an academic article with referencing, do not be put off, there are enough other topics that you can write about without those academic constraints. For those of you who have experience in academic writing, take the plunge and send us your work. You will be surprised at just how good it looks in print! So have a go and send all your ideas and submissions to: jessinchbarna@ googlemail.com. 